\title{
Article
}

\section{Graphene quantumdots: In the crossroad of graphene, quantum dots and carbogenic nanoparticles}

Kelarakis, A

Available at http://clok.uclan.ac.uk/14914/

Kelarakis, A ORCID: 0000-0002-8112-5176 (2015) Graphene quantumdots: In the crossroad of graphene, quantum dots and carbogenic nanoparticles. Current Opinion in Colloid \& Interface Science, 20 (5-6). pp. 354-361. ISSN 1359-0294

It is advisable to refer to the publisher's version if you intend to cite from the work. http://dx.doi.org/10.1016/j.cocis.2015.11.001

For more information about UCLan's research in this area go to http://www.uclan.ac.uk/researchgroups/ and search for < name of research Group>.

For information about Research generally at UCLan please go to http://www.uclan.ac.uk/research/

All outputs in CLoK are protected by Intellectual Property Rights law, including Copyright law. Copyright, IPR and Moral Rights for the works on this site are retained by the individual authors and/or other copyright owners. Terms and conditions for use of this material are defined in the policies page.

\section{CLoK}

Central Lancashire online Knowledge www.clok.uclan.ac.uk

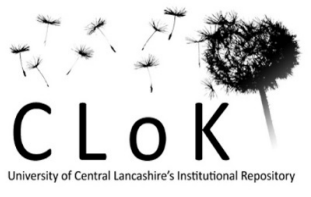




\title{
Graphene quantum dots: in the crossroad of graphene, quantum dots and carbogenic nanoparticles
}

\author{
Antonios Kelarakis \\ Centre for Materials Science, School of Physical Sciences and Computing, University of Central \\ Lancashire, Preston PR12HE, U.K. \\ Email:akelarakis@uclan.ac.uk
}

\begin{abstract}
The scientific and technological importance of graphene quantum dots (GQDs) is directly related to their nanoscopic nature that endows remarkable photo-physical properties and colloidal stability in a variety of solvents. GQDs combine characteristics arising from their graphitic structure, their carbogenic origin and their quantum nature. They are considered as the environmentally benign alternatives of heavy metal based quantum dots, given that not only are they synthesized following green strategies, but they also exhibit minimal toxicity. GQDs are systematically explored in printing, energy harvesting, bioimaging, catalysis, optoelectronics and sensing applications.
\end{abstract}

\section{Introduction}


As their name "graphene quantum dots" (GQDs) implies, this emerging class of nanomaterials is placed in the crossroad of the electronically conductive highway of graphene, the photoluminescent (PL) avenue of quantum dots and the green, widely unexplored, pathway of carbogenic nanoparticles. The term "dot" refers to their nanoscopic nature that endows remarkable optical properties ${ }^{[1-4]}$ comparable to the best performing fluorescent materials. Due to their advanced PL properties, GQDs are systematically explored in solar cells, optoelectronics, catalysis, cell imaging and sensing.

While pure graphene (an atomic monolayer of carbon perfectly arranged in a honeycomb conformation) is not emissive, energy band gaps can be engineered by introducing structural defects $^{[5 * \cdot]}$ in a graphene plane or via its quantum confinement towards 1D (graphene nanoribbons ${ }^{[6]}$ ) or OD (GQDs) geometries. The PL behavior of GQDs can be tuned from ultraviolet to near infrared depending on the size, shape, edge effects, functional groups, heteroatom doping and $\mathrm{sp}^{2}$ carbon fraction.

In addition to their supreme PL behavior, GQDs show a number of attractive characteristics. First, they exhibit minimal toxicity for humans and the environment (see section 5.1), in contrast to their heavy metal based counterparts. Second, they are synthesized in bulk from abundant starting materials by low-cost strategies. Third, because GQDs are dispersible in water and organic solvents, they are readily integrated into standard industrial manufacturing.

The progress achieved so far with respect to the synthesis, characterization and applications of GQDs has been reviewed in a number of recent reports ${ }^{[1-4]}$. Here we attempt to present a compact synopsis of the current state of understanding in GQDs highlighting their graphitic structure, their carbogenic origin and their quantum nature.

Strictly speaking, GQDs refer to nano-fragments of atomically thick graphene, however the term is also used to describe few-layer thick nanodisks of graphite, graphite oxide quantum dots and partially reduced graphite oxide quantum dots. We note that oftentimes in literature, the terms carbogenic nanoparticles, carbon dots, carbon quantum dots and GQDs are used interchangeably ${ }^{[7]}$. Here we refer to carbogenic nanoparticles (C-dots) as a broad class of highly PL materials that includes GQDs, along with other types of carbon-rich particles with partially or predominantly amorphous cores. 


\section{The graphitic structure}

Monodispersed GQDs are prepared by all-organic synthesis starting from polyphenylene dendrimers $^{[8]}$, alkyne containing reactive molecules ${ }^{[9]}$ or via self-assembly and carbonization of unsubstituted hexa-peri-hexabenzocoronene precursors ${ }^{[10]}$. Alternatively, well-defined GQDs derived by ring opening of fullerenes that are strongly adsorbed to Ru metal terraces, undergo fragmentation and gasification at elevated temperatures to generate surface-stabilized carbon clusters that coalesce towards uniform structures ${ }^{[11]}$.

A distinct advantage of GQDs is that they can be produced in bulk based on the chemical oxidation of carbon-rich sources such as coal ${ }^{[12 \cdot]}$, $\operatorname{carbon}_{\text {black }}{ }^{[13]}$, graphite ${ }^{[14]}$, carbon fibers $(\mathrm{CF})^{[15]}, \mathrm{CNT}^{[16]}$ and fullerenes ${ }^{[17]}$. Alternatively, electrooxidation of $\mathrm{CNTs}^{[18]}$ and graphite ${ }^{[19]}$ leads to the formation of hydroxyl and oxygen radicals (produced by the electrolysis of the solvent) that attack the honeycomb lattice on defect and edge sites, facilitating the release of GQDs.

GQDs with relatively narrow size distribution between 1 to $4 \mathrm{~nm}$ and a predominant zigzag edge structure are derived by chemical oxidation and cutting of microsized pitch based $\mathrm{CF}^{[15]}$. The TEM images (Fig. 1a) reveal a lattice spacing of $0.24 \mathrm{~nm}$, while the Atomic Force Microscopy (AFM) topographic heights (Fig. 1b) indicate that GQDs are composed by 1-3 graphene layers. X-ray Photoelectron Spectroscopy (XPS) patterns of QGDs and CF (Fig. 1c-e) display the C1s peak at $284.8 \mathrm{eV}$ and the O1s peak at $532 \mathrm{eV}$ and suggest a higher oxygen content for GQDs. Analysis of the C1s peak of CF and QGDs (Fig. 1d and 1e, respectively) reveals the enhanced presence of C $=\mathrm{C}, \mathrm{C}-\mathrm{O}, \mathrm{C}=\mathrm{O}$ bonds and $-\mathrm{COOH}$ groups on the surface of GQDs.

The Raman spectra of GQDs and CF (Fig. 1f) are governed by the two characteristic G and D bands of the graphitic and disordered carbon, respectively. The G peak is attributed to the $E_{2 g}$ phonon at the Brillouin center, while the $\mathrm{D}$ peak corresponds to the breathing mode of $\mathrm{sp}^{2}$ centers and becomes active in proximity to an $\mathrm{sp}^{3}$ defect ${ }^{[20]}$. The relative intensity of the $\mathrm{D}$ over $\mathrm{G}$ band, $\mathrm{I}_{\mathrm{D}} / \mathrm{I}_{\mathrm{G}}=0.9$, implying a pronounced graphitization degree. Fourier transform infrared (FTIR) spectra (Fig. 1g) provide further evidence for the evolution of $-\mathrm{OH}, \mathrm{C}=\mathrm{O}, \mathrm{C}=\mathrm{C}, \mathrm{C}-\mathrm{H}$ and $\mathrm{C}-\mathrm{O}$ in GQDs. Those groups function as a self-passivating layer that render GQDs dispersible in a variety of solvents. The XRD profile of GQDs (Fig. 1h) shows a broad peak at $4.0 \AA$ compared to a sharp 
peak at 3.6 $\AA$ for the initial CF. The larger interlayer spacing found for GQDs is consistent with the generation of oxygen-containing groups during oxidation and exfoliation of CF.
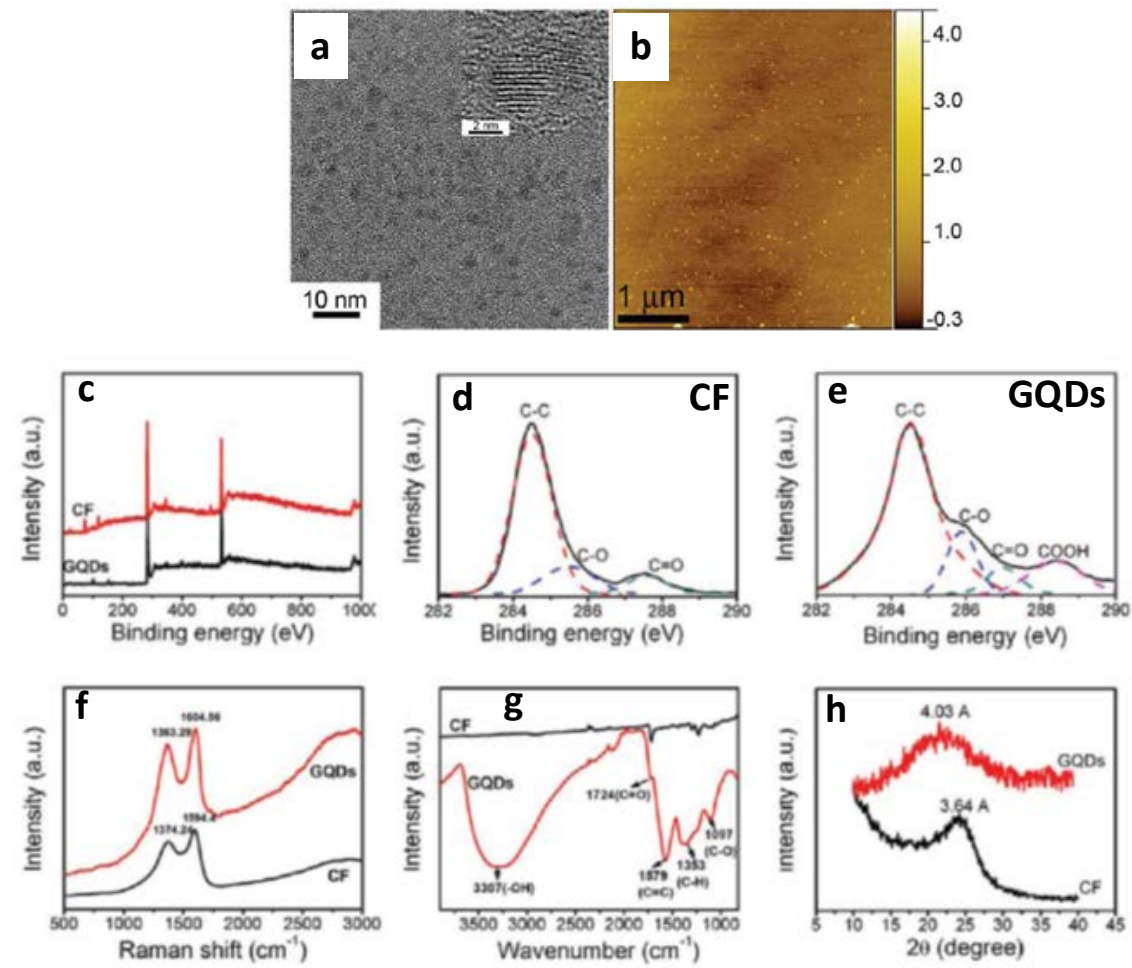

Figure 1. GQDs released from carbon fibers (CF) via chemical oxidation; (a) TEM image, (b) AFM image, (c,d,e) XPS spectra, (f) Raman spectra, (g) FTIR spectra and (h) XRD patterns. Reprinted with permission from ref. 15. Copyright 2012 American Chemical Society.

\section{The quantum nature and its limitations}

Graphite oxide (GO) consists of a graphene plane bearing numerous oxygen-rich groups, a situation that can be seen as $\mathrm{sp}^{2}$ carbon islands dispersed within a $\mathrm{sp}^{3}$ matrix. The PL spectra of aqueous suspensions of GO can be deconvoluted into two Gaussian bands ( $\mathrm{I}_{\mathrm{p} 1}$ and $\mathrm{I}_{\mathrm{p} 2}$ in Fig. 2 a,b,c), suggesting the parallel action of two distinct emissive contributions ${ }^{[21 \cdot \bullet]}$. The relative intensities of those contributions significantly change during the photothermal reduction (via xenon lamp irradiation) of GO that results in a dramatic increase of the $\mathrm{sp}^{2}$ carbon content from $25 \%$ of the starting GO (derived via a modified Hummers method) all the way to $69 \%$ after $3 \mathrm{~h}$ exposure. 


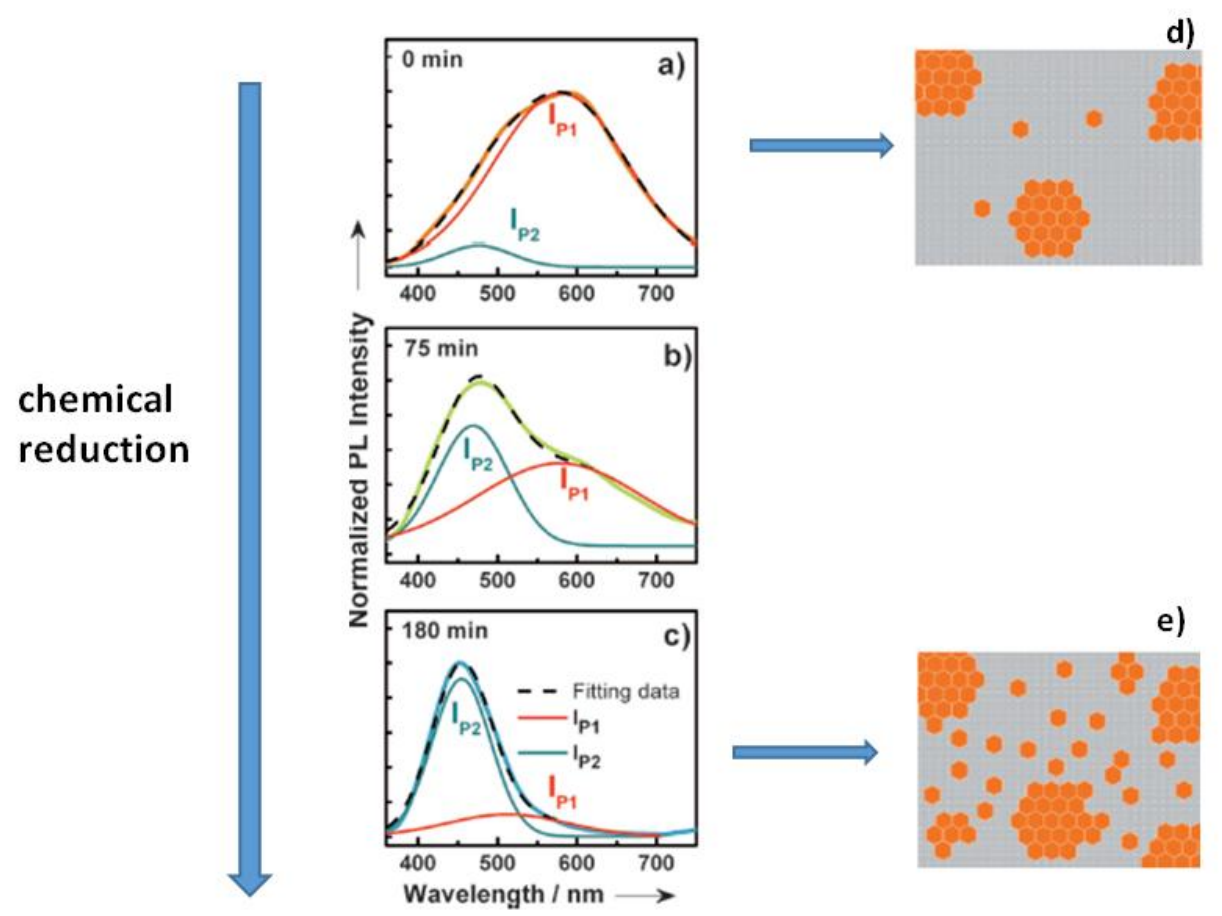

Figure 2. PL spectra of aqueous suspensions of GO at reduction times; (a) O min, (b) $75 \mathrm{~min}$ and (c) $180 \mathrm{~min}$. Note that each plot can be fitted using two Gaussian curves. ( $\mathrm{d}$ and e). Graphics showing the birth of small and isolated sp ${ }^{2}$ islands during the gradual chemical reduction of GO. Reprinted with permission from ref. 21. Copyright 2012 WileyVCH Verlag GmbH \& Co.

Nanoscale morphological characterization (via Scanning Tunnelling Microscopy and Transmission Electron Microscopy) reveals that the gradual chemical reduction of GO preferably proceeds through the removal of oxygen atoms positioned far from $\pi$-conjugated domains. This trend favours the formation of new, small and isolated $\mathrm{sp}^{2}$ islands, and leaves pre-existing $\mathrm{sp}^{2}$ clusters essentially unaltered (Fig. 2 d,e). As the reduction treatment proceeds, the $\mathrm{I}_{\mathrm{p} 2}$ Gaussian band is systematically enhanced, suggesting that it stems from the $\mathrm{sp}^{2}$ carbon. Notably, $\mathrm{I}_{\mathrm{p} 2}$ shifts to lower wavelengths, consistent with quantum confinement effects expected for the numerous small $\mathrm{sp}^{2}$ domains. At the same time, the initially predominant $\mathrm{I}_{\mathrm{p} 1}$ component monotonously decreases during deoxygenation, an effect consistent with the gradual elimination of the defect states. 
Early studies indicated that the optical properties in carbon-based materials containing different configurations of $\mathrm{sp}^{2}$ and $\mathrm{sp}^{3}$ sites are determined by the $\pi-\pi^{*}$ electronic levels and the band gap varies inversely with the $\mathrm{sp}^{2}$ cluster $\operatorname{size}^{22}$. Recent theoretical studies indicated that the HUMOLUMO band gap in GQDs can be tuned from $7 \mathrm{eV}$ for a single benzene ring to $2 \mathrm{eV}$ for $20 \pi$ conjugated aromatic rings ${ }^{[23]}$, in analogy to semiconductor quantum dots.
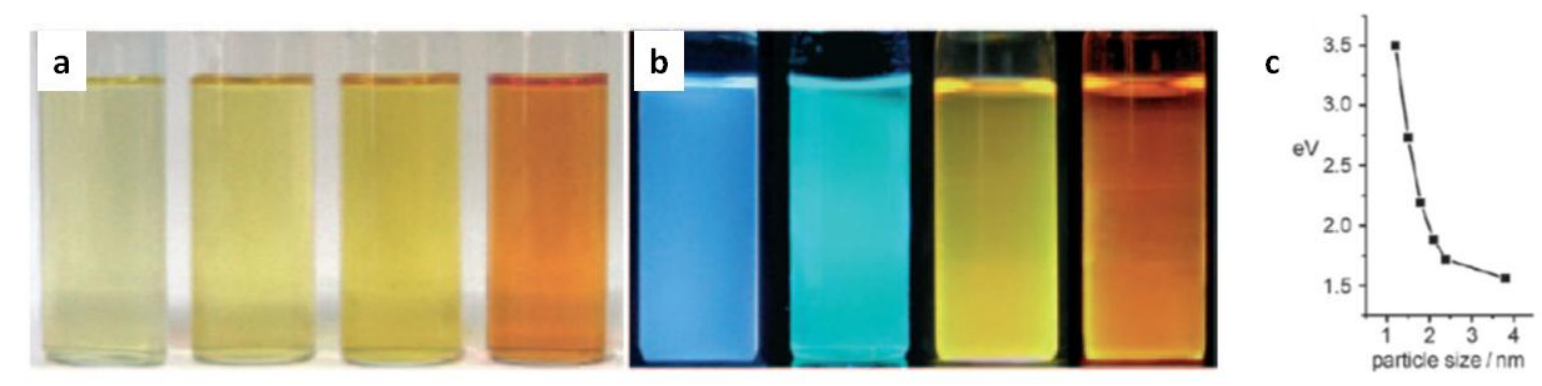

Figure 3. Images of aqueous dispersions of different sized GQDs in water under (a) white and (b) UV illumination (b). (c) Dependence of the band gap as a function of the GQD size. Reprinted with permission from ref 24. Copyright 2010 Wiley-VCH Verlag GmbH \& Co.

To that end, alkali assisted electrooxidation of graphite leads to a mixture of different sized GQDs that can be separated to relatively uniform particle fractions by column chromatography ${ }^{[24]}$. The aqueous dispersions of those fractions under white and UV light are shown in Fig. 3a and 3b, respectively, providing direct evidence on their quantum nature. Figure 3c shows that the band gap of GQDs decreases as the GQD size increases. In addition, hydrogen plasma treatment had no detectable effect on their PL spectra, confirming that the optical properties are attributed to quantum confinement and not to the presence of surface oxygen.

In another study, a series of well-defined GQDs with sizes from 2 to $10 \mathrm{~nm}$ was produced via amidative cutting of pre-oxidized graphite flakes ${ }^{[25]}$. Interestingly, their aqueous dispersions under UV light display a variety of colors from blue to brown, in a manner that critically depends on their size (Fig. 4a). The excitation matrices (a set of excitation spectra, each one collected at a fixed emission wavelength) of the GQDs with sizes 2,4,7, and $10 \mathrm{~nm}$ are shown in Figures 4b-e. The two peaks observed in each data set were attributed to the electronic transition from the ground state to the lowest and the second lowest excited state, respectively; both peaks systematically red shift upon increasing the particle size, consistent with quantum confinement effects. 

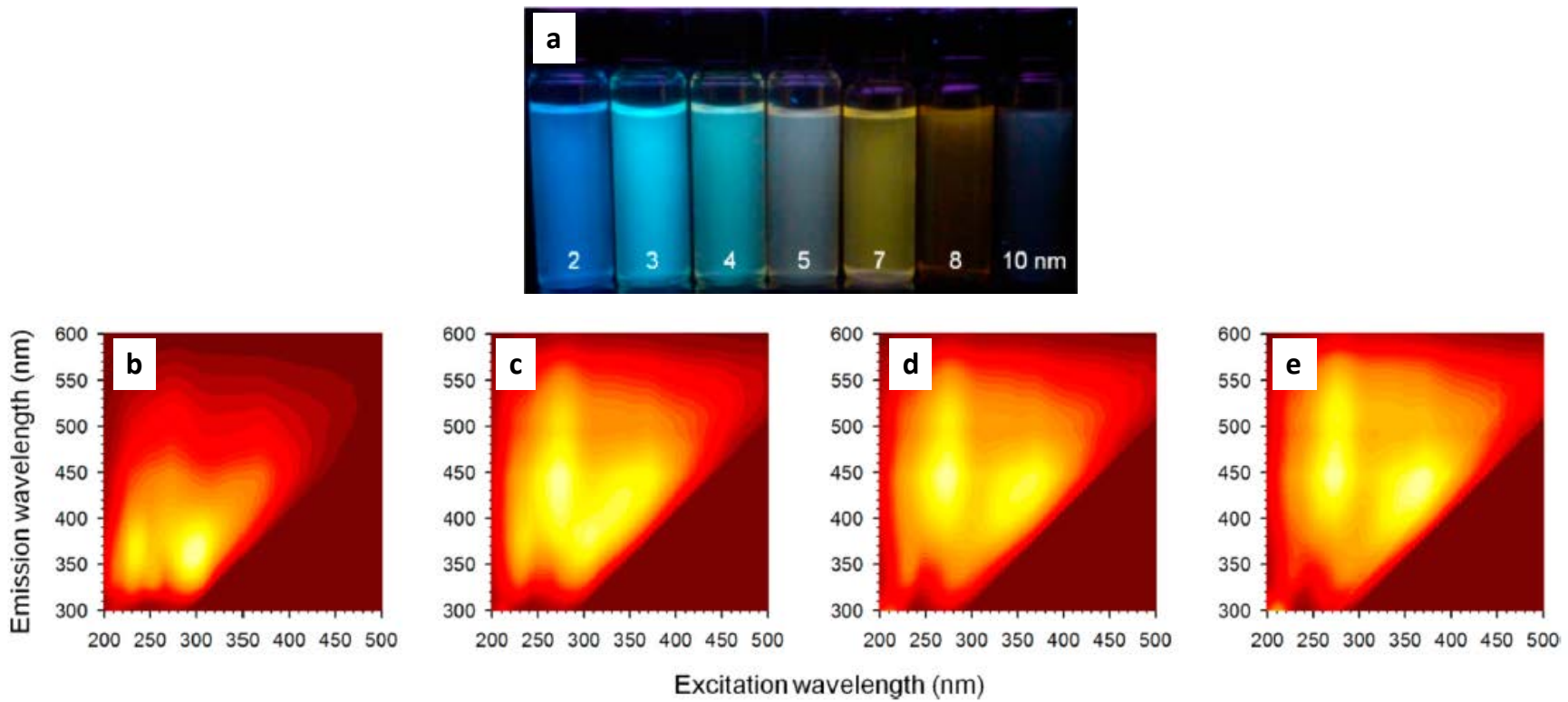

Figure 4. (a) Images of aqueous dispersions of different sized GQDs in water under UV illumination. The captions denote the size of GQDs in nm. (b-e) Emission matrices of aqueous dispersion of GQDs with sizes 2, 4,7 and $10 \mathrm{~nm}$, respectively. Reprinted with permission from ref 25. Copyright 2014 American Chemical Society.

There is a growing body of evidence to suggest that PL in GQDs cannot be explained in terms of quantum confinement alone and that pronounced contributions arise from edge effects, the presence of functional groups, heteroatom doping and the fraction of $\mathrm{sp}^{2}$ domains. For example, the emission wavelength of GQDs continuously shifts to higher values as a function of the -OH and -COOH coverage to edge carbon atoms (Fig. 5). In particular, for cis-coronene (G4) $\mathrm{E}_{\mathrm{m}}=572.4$ $\mathrm{nm}$, but approaches $723.3 \mathrm{~nm}$ for $100 \%$ edge coverage with $-\mathrm{OH}^{[26 \cdot]}$. When two $-\mathrm{OH}$ groups are conjugated on the basal plane of G4, a substantial red-shift is observed, resulting in $E_{m}=746.1 \mathrm{~nm}$. Experimental data confirm that green GQDs, when subjected to mild chemical reduction, become blue nanoemitters ${ }^{[27]}$, in agreement with the trends shown in Fig. 5.

Graphitic N-doping on the green G4 substantially lowers the band gap to the extent that the particles become non emissive ${ }^{[26 \bullet}$. Pyridinic $\mathrm{N}$-doping causes a blue shift, so that $12.5 \% \mathrm{~N} / \mathrm{C}$ ratio shifts the emission from 572.4 to $550.3 \mathrm{~nm}$. Introduction of pyrrolic $\mathrm{N}$ atoms (N/C ratio $=9.09 \%$ ) at the edged five-membered rings of GQDs causes blue shift from 624.4 to $575.5 \mathrm{~nm}^{[26 \cdot]}$. 


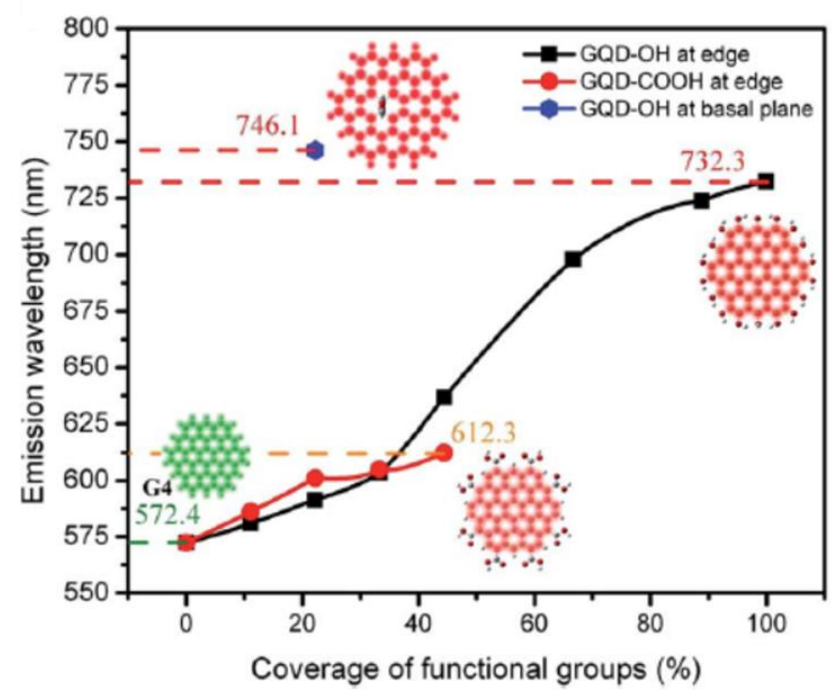

Figure 5. Emission wavelength of oxidized cis-coronene (G4) as a function of the coverage of -OH and - $\mathrm{COOH}$ groups. Reprinted with permission from ref 26. Copyright 2014, Royal Society of Chemistry.

\section{The common carbogenic origin}

Carbogenic nanoparticles, commonly described as C-dots, are spherical nanoemitters consisting of a partially or predominantly amorphous core. In carbon materials that combine carbons with different hybridizations, it should be noted that when $\mathrm{sp}^{3}$ carbons are used to bridge two benzene rings, the emission shifts to lower wavelengths. For example, anthracene is composed of two benzene rings connected via two sp ${ }^{2}$ carbons and emits at $435 \mathrm{~nm}$ (Fig. 6 a), however when two benzene rings are connected via two or six $\mathrm{sp}^{3}$ carbons they emit at 256.9 and $241.9 \mathrm{~nm}$, respectively ${ }^{[26 \cdot]}$ (Fig. 6 b and c).

a

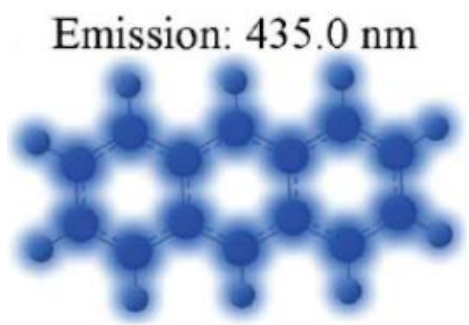

b

Emission: $256.9 \mathrm{~nm}$

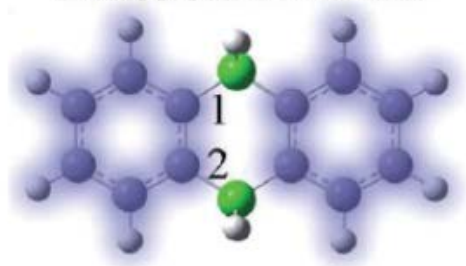

C

Emission: $241.9 \mathrm{~nm}$

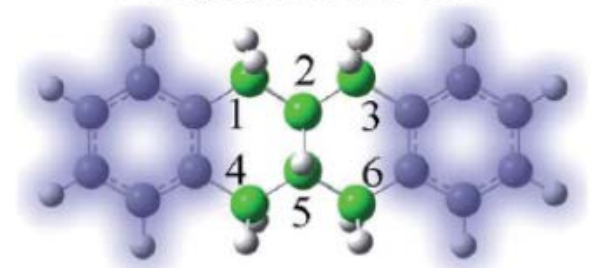

Figure 6. Two benzene rings joined via (a) two sp ${ }^{2}$ carbons, (b) two $\mathrm{sp}^{3}$ carbons and (c) six sp $\mathrm{s}^{3}$ carbons. Reprinted with permission from ref 26. Copyright 2014, Royal Society of Chemistry. 
C-dots are typically derived via hydrothermal or pyrolytic treatment of carbon-rich precursors including renewable materials such as biomass ${ }^{[28]}$, fruit juices ${ }^{[29]}$ and carbohydrates ${ }^{[30]}$. The TEM images (Fig. 7a) of the polysaccharide-derived C-dots do not show any discernible lattice fringes, indicating the amorphous nature of the core ${ }^{[31]}$. TEM and AFM (Fig. 7b) both suggest particle diameters between $2-5 \mathrm{~nm}$. The XRD pattern shows only a broad peak at $2 \theta=23.1^{\circ}$ (Fig. $7 \mathrm{c}$ ), consistent with the amorphous carbon phase. The FTIR spectrum of C-dots indicates the presence of surface $\mathrm{C}-\mathrm{H}, \mathrm{C}=\mathrm{C}, \mathrm{C}=\mathrm{O}, \mathrm{C}-\mathrm{O}$ and $-\mathrm{OH}$ groups that impart solubility in various solvents. XPS (Fig. 7e) reveals the presence of $\mathrm{C}$ and $\mathrm{O}$, while the XPS C1s spectrum in Fig. $7 \mathrm{f}$ shows peaks at 284.3, 285.7 and $288.0 \mathrm{eV}$, corresponding to $\mathrm{C}=\mathrm{C}-\mathrm{C}, \mathrm{C}-\mathrm{O}$ and $\mathrm{C}=\mathrm{O}$ groups, respectively.

Figure 7. C-dots hydrothermally derived from a polysaccharide: (a) TEM, (b) AFM image (inset displays the height profile along the line), (c) XRD, (d) FTIR, (e) XPS spectra (e) and XPS C1s (f) spectra of C-Dots. Reprinted with
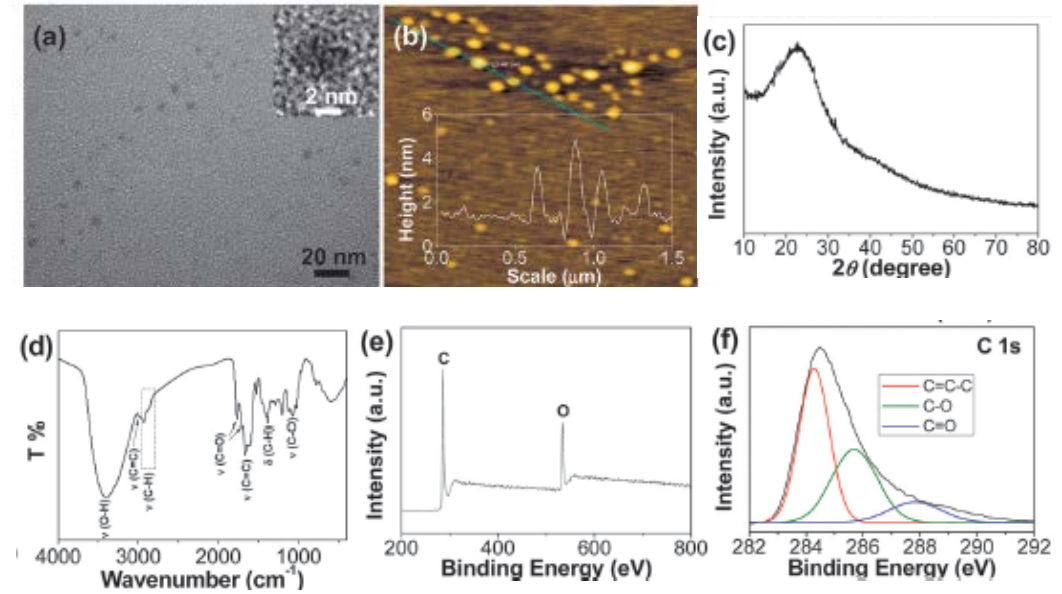

permission from ref 31. Copyright 2013, Royal Society of Chemistry.

Despite their pronounced structural differences, C-dots ${ }^{[32]}$ and GQDs ${ }^{[14]}$ share common PL patterns and, as the excitation wavelength increases, the emission peak is displaced to longer wavelengths and a less intense signal is recorded (Fig. 8).

It has been pointed out that defects in graphene sheets related to the presence of $\mathrm{sp}^{3}$ carbons are structurally no different from defects on the surface of C-dots and this observation explains the similarities between the PL characteristics in C-dots and GQDs ${ }^{\left[33^{\circ}\right]}$. We note that for GQDs, the defect-derived PL contributions can be substantially stronger compared to emissions stemming from quantized $\mathrm{sp}^{2}$ islands ${ }^{\left[33^{\circ}\right]}$. A recent study suggests that the green PL centers encountered in 
both C-dots and GQDs originate from emissive edge states due to the combination of carboxyl groups and carbonyl groups ${ }^{[34]}$.

Figure 8. PL emission at different excitation wavelengths of aqueous dispersions containing: (a) C-dots derived by
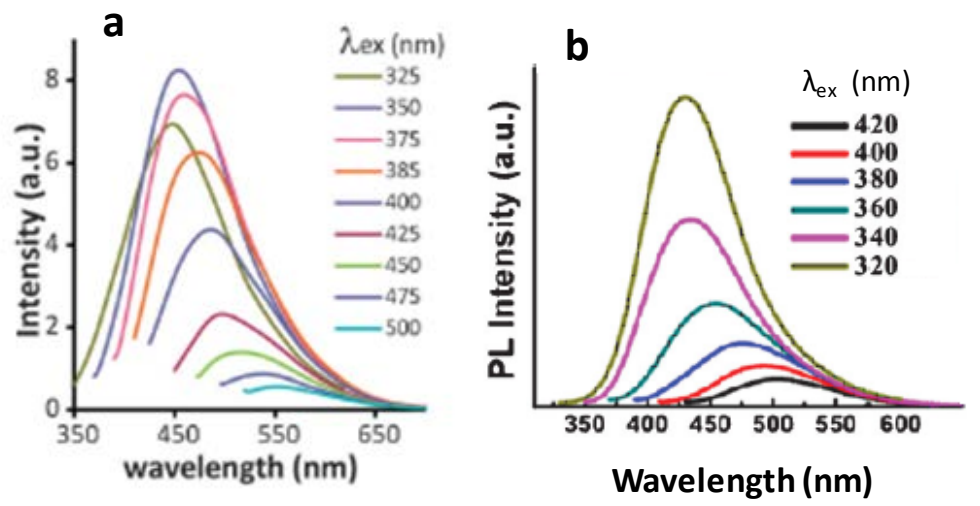

caramelization of poly(ethylene glycol) and (b) GQDs derived hydrothermally from graphene sheets. Reprinted with permission from ref 32 (Copyright 2011, Royal Society of Chemistry) and 14 (Copyright 2010 Wiley-VCH Verlag GmbH \& Co), respectively.

It has been proposed that the PL behavior in C-dots arises from the radiative recombinations of surface confined photogenerated electron and holes pairs; the role of passivation agents is to stabilize the surface sites and improve the PL intensity ${ }^{[35]}$. Similar effects have been observed in GQDs $^{[36]}$, further confirming the common PL origins for GQDs and C-dots.

\section{Applications}

\subsection{Cell imaging}

The viability of HeLa cells incubated for $24 \mathrm{~h}$ with GQDs at concentration up to $500 \mathrm{mg} \mathrm{mL}^{-1}$ exceeds $95 \%{ }^{[37]}$ (Fig. 9a), suggesting that GQDs exhibit minimal cytotoxicity. Moreover, the cells display bright green PL, indicating a high degree of GQDs uptake (Fig. 9b). By virtue of their small hydrodynamic ratio and their low nonspecific protein adsorption, GQDs are promising candidates for bioimaging. Following intravenous administration GQDs remained highly fluorescent in vivo and followed the urine excretion ${ }^{[38]}$. GQDs that emit in the near IR have been recently reported ${ }^{[39]}$ and carry the promise to circumvent issues related to cell autofluorescence and undesired photo-damage of sensitive tissue. 

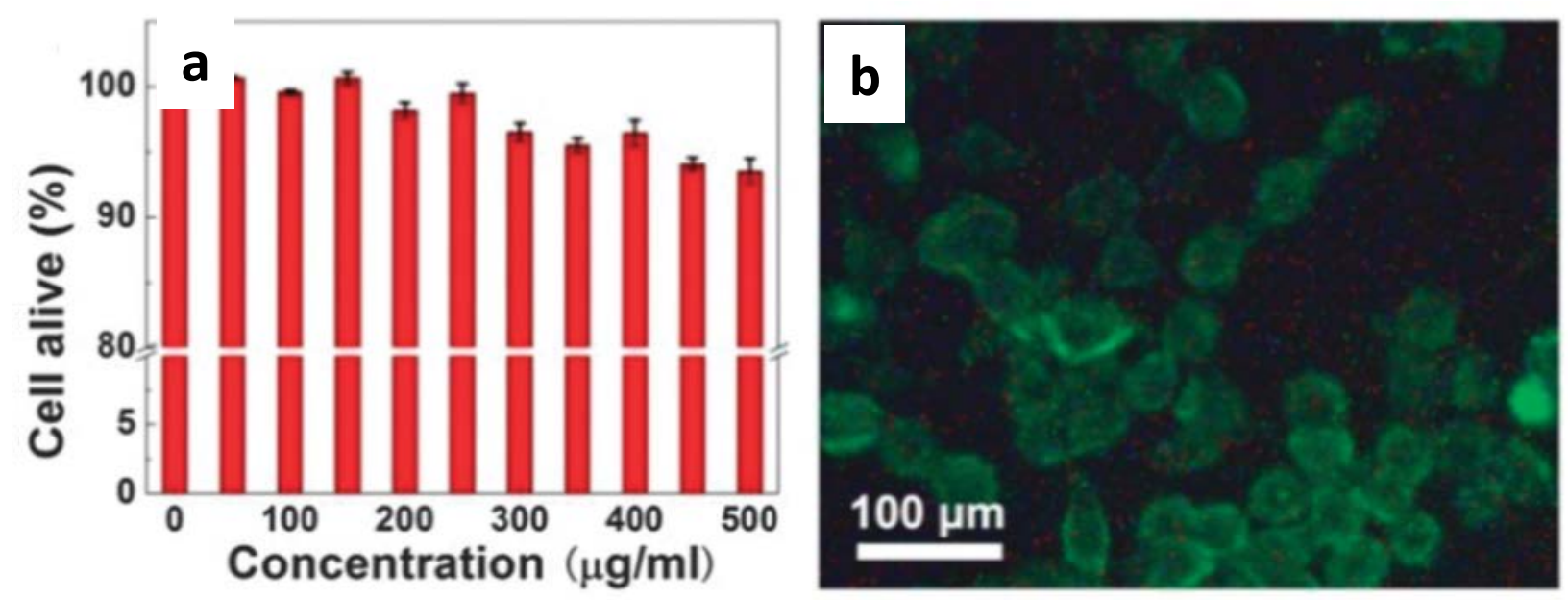

Figure 9. (a) Viability of Hela cells incubated for 24h with GQDs. (b) Confocal fluorescence microscope PL image of Hela cells incubated with $100 \mu \mathrm{g} / \mathrm{mL}$ GQDs $(\lambda \mathrm{ex}=350 \mathrm{~nm})$. Reprinted with permission from ref 37 . Copyright 2015, Royal Society of Chemistry.

\subsection{Catalysis and photocatalysis}

The slow kinetics associated with the oxygen reduction reaction (ORR) impose barriers to the commercialization of fuel cells, given that the commonly used carbon supported Pt catalysts are expensive and prone to methanol poisoning. Recent work ${ }^{[40]}$ indicates that the catalytic activity of $\mathrm{N}$-doped GQDs is comparable to that of carbon supported Pt, with the added advantage that the former materials exhibit remarkable tolerance against methanol crossover, a situation commonly encountered in fuel cells. Boron and nitrogen co-doped GQDs deposited on graphene also exhibit improved ORR catalytic activity, showing $15 \mathrm{mV}$ more positive onset potential and similar levels of current density compared to standard catalysts ${ }^{[41]}$.

As shown in Fig. 10, GQDs based composite materials (combined with $\mathrm{TiO}_{2}, \mathrm{Fe}_{2} \mathrm{O}_{3}, \mathrm{Ag}_{3} \mathrm{PO}_{4}$ ) exhibit significant photocatalyic activity for the decomposition of dyes, organic compounds and toxic gasses $^{[24,42,43]}$. The effect reflects the synergistic action of the electron donating properties of GQDs, their upconverted luminescence and the extensive $\pi$ - $\pi$ stacking between the aromatic rings of the organic molecules and the catalysts. 

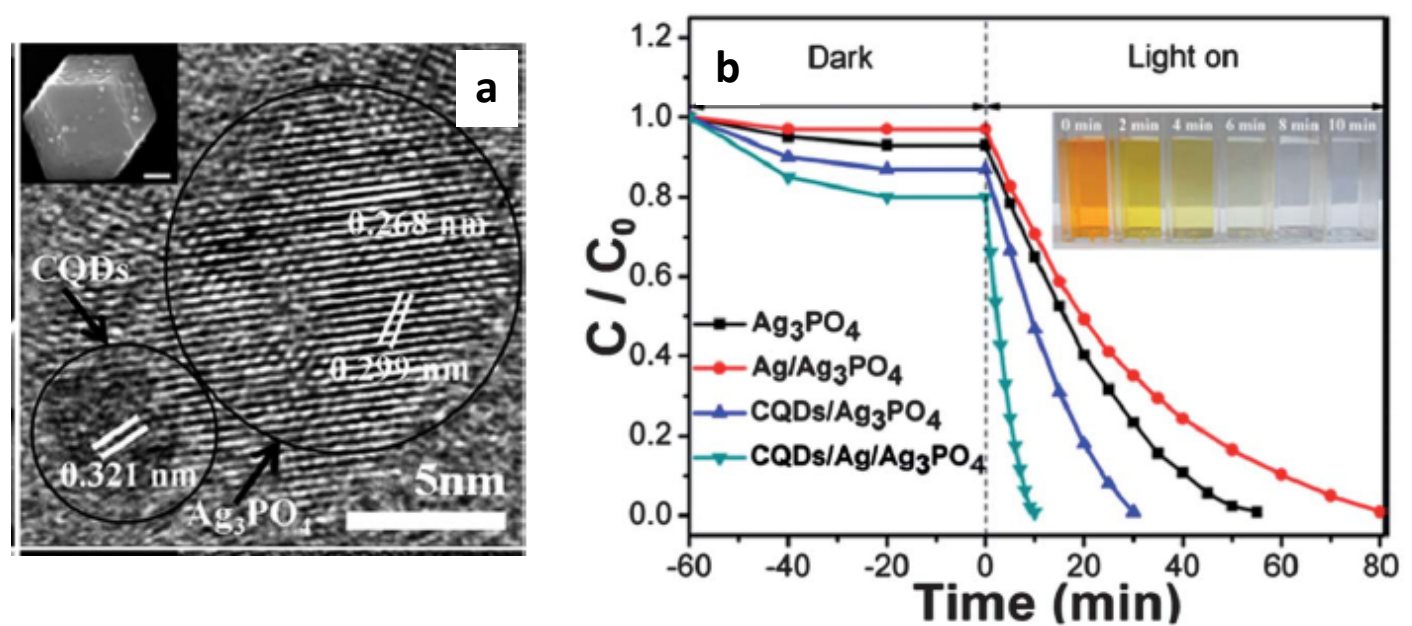

Figure 10. (a) TEM Images of GQDs/ $\mathrm{Ag}_{3} \mathrm{PO}_{4}$ complex photocatalyst. (b) Decomposition rate of methyl blue (MB) in the presence of $\mathrm{Ag}_{3} \mathrm{PO}_{4}, \mathrm{Ag} / \mathrm{Ag}_{3} \mathrm{PO}_{4}$, GQDs/ $\mathrm{Ag}_{3} \mathrm{PO}_{4}$ and GQDs/Ag/ $/ \mathrm{Ag}_{3} \mathrm{PO}_{4}$ under visible-light. The inset shows the color changes of the MB solutions corresponding to the degradation times of $0,2,4,6,8,10$ min over GQDs/Ag/Ag ${ }_{3} \mathrm{PO}_{4}$. Reprinted with permission from ref 43. Copyright 2012, Royal Society of Chemistry.

\subsection{Sensing applications}

The PL properties of GQDs often exhibit selective quenching in the presence of specific ions or molecules, providing a platform for the development of high performance sensors ${ }^{[44]}$. In water, 2,4,6-trinitrotoluene (TNT) interacts via $\pi-\pi$ stacking with GQDs in a manner that suppresses PL emission via fluorescence resonance energy transfer (FRET) ${ }^{[45]}$. This system allows ultrasensitive detection of the explosive molecules with a detection limit down to $0.495 \mathrm{ppm}$ TNT using only 1 $\mathrm{mL}$ of GQDs solution.

In another application, $\mathrm{Eu}^{3+}$ ions are able to coordinate the carboxylate ions on the surface of GQDs leading to PL quenching through energy-transfer or electron-transfer processes. Because phosphate ions (Pi) display strong affinity to $\mathrm{Eu}^{3+}$ they break down the GQDs-Eu ${ }^{3+}$ complexes (Fig. 11a) resulting in a substantial enhancement of PL intensity ${ }^{[46]}$. The sensor is highly selective to Pi ions (Fig. 11b). 
Figure 11. (a) Phosphate (Pi) detection based on the antagonistic interaction of $\mathrm{Eu}^{3+}$ with GQDs or Pi molecules. (b)
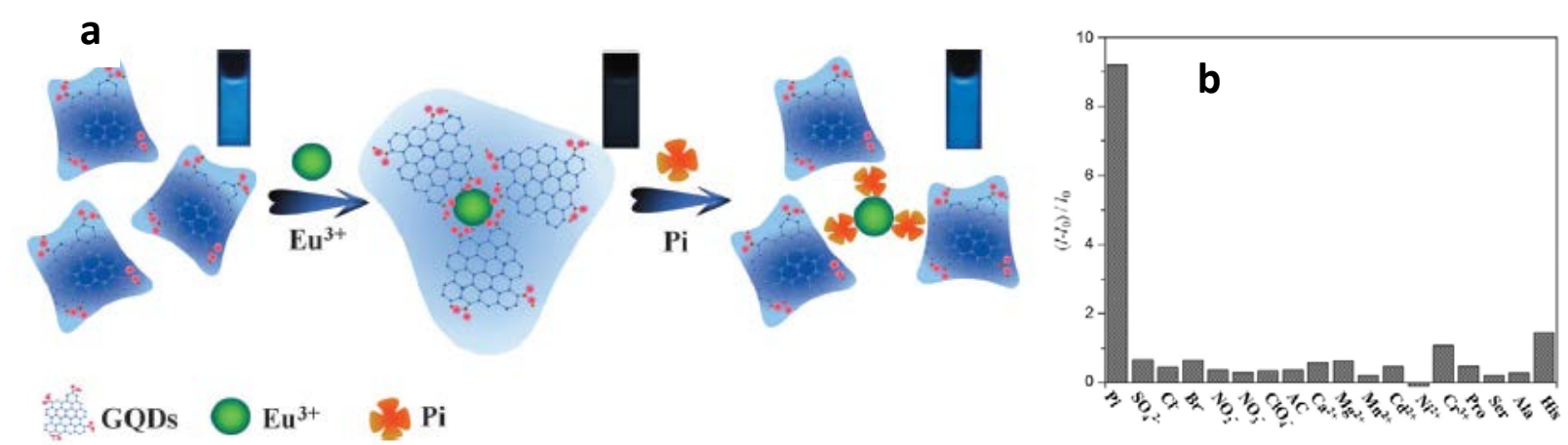

Selectivity of the PL platform for Pi detection compared to other substances. Reprinted with permission from ref 46. Copyright 2013 Wiley-VCH Verlag GmbH \& Co.

\subsection{Energy harvesting}

GQDs are ideal candidates as sensitizers in solar cells given that their absorption edge extends up to $900 \mathrm{~nm}$ and they exhibit one order of magnitude higher absorbance compared to standard metal complexes. In the first proof-of-concept demonstration ${ }^{[8]}$, the low current density observed pointed to the low affinity of GQDs and the $\mathrm{TiO}_{2}$. In a recent study, N-GQDs/TiO 2 hierarchical microspheres were applied in dye-sensitized solar cells, showing open circuit voltage, fill factor and power conversion efficiency $0.46 \mathrm{~V}, 43 \%$ and $0.13 \%$, respectively ${ }^{[47]}$.

In addition, GQDs have been explored as electron acceptors in order to improve the performance in bulk heterojunction (BHJ) solar cells. Incorporation of GQDs in a solar cell assembly ITO/PEDOT:PSS/P3HT:GQDs/Al [ITO, PEDOT, PSS, and P3HT stand for indium tin oxide, poly(3,4-ethylenedioxythiophene), poly(styrenesulfonate), and poly(3-hexylthiophene)] dramatically improves the power conversion efficiency (PCE) and short-circuit current $\left(\mathrm{J}_{\mathrm{sc}}\right)^{[48]}$ (Fig. 12). This behavior points to the fact that GQDs afford large p-n interfaces for charge separation. 
Partially reduced GQDs derived by graphite oxide dots were found to exhibit optimal performance compared to their highly oxidized and fully reduced counterparts ${ }^{[99]}$. This behavior indicates that the overall performance reflects an interplay between enhanced light absorptivity in the highly oxidized particles that increases $\mathrm{J}_{\mathrm{sc}}$ and improved conductivity in the fully reduced particles that increases the fill factors (FF). Overall, incorporation of the partially reduced GQDs resulted in PCE of $7.6 \%$ compared to $6.7 \%$ for the GQD-free device.

a

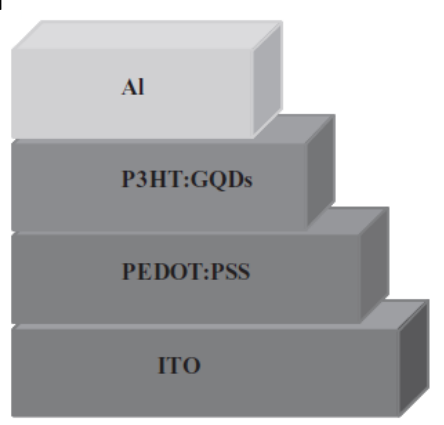

b

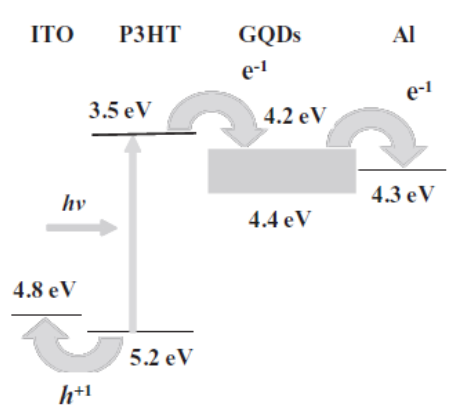

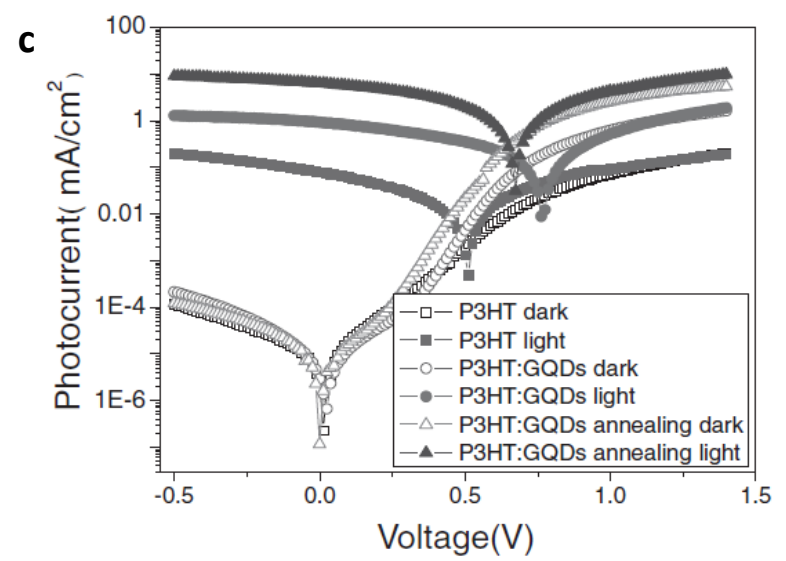

Figure 12. Schematic (a) and energy band (b) diagrams of the ITO/PEDOT:PSS/P3HT:GQDs/ Al device. c) $J-V$ characteristic curves for the ITO/PEDOT:PSS/P3HT/Al, ITO/PEDOT:PSS/ P3HT:GQDs/Al and ITO/PEDOT:PSS/P3HT: GQDs/Al devices after annealing at $140^{\circ} \mathrm{C}$ for $10 \mathrm{~min}$. Reprinted with permission from ref 48. Copyright 2011 John Wiley and Sons.

\section{Conclusions}

Historically, the rise of GQDs coincides with the rise of graphene and the two newcomers in the carbon family are expected to act synergistically in sophisticated devices. It is, nevertheless, clear 
that structural defects and quantum confinement are essential for the performance of GQDs, but are detrimental for the electronic conduction of graphene.

GQDs are naturally occurring nanoparticles that can be released by coal, carbon fibers, graphite and other carbon sources. It is perhaps surprising that GQDs have been discovered only recently, two decades after the development of conventional quantum dots. Due to their strong optical absorption and excitation wavelength dependent PL emission that is not prone to photobleaching, GQDs can match and outperform highly engineered, albeit toxic, counterparts in various applications. Moreover, given that they can be produced in bulk by green methods, GQDs showcase a viable example of sustainable chemistry.

GQDs are novel nanoemitters that seem to have it all: bright fluorescence, facile and low cost preparation, minimal toxicity, supreme structural and colloidal stability. During the last years, GQDs have undoubtedly demonstrated their great potential in a series of highly demanding applications. Within the next decade, the challenge for GQDs is to open new horizons and allow new applications, currently inaccessible with excising technologies, in the fields of theranostics, catalysis, energy conversion and optical diodes.

\section{References and recommended reading $\bullet, \bullet$}

[1] Lingling L, Gehui W, Guohai Y, Juan P, Jianwei Z, Jun-Jie Z. Focusing on luminescent graphene quantum dots: current status and future perspectives. Nanoscale 2013;5:4015-4039.

[2] Bacon M, Bradley SJ, Nann T. Graphene quantum dots. Part Part Syst Charact 2014; 31:415428.

[3] Zheng XT, Ananthanarayanan A, Luo KQ, Chen P. Glowing graphene quantum dots and carbon dots: properties, syntheses, and biological applications. Small 2015; 11:1620-1636.

[4] Shen J, Zhu Y, Yang X, Li C. Graphene quantum dots: emergent nanolights for bioimaging, sensors, catalysis and photovoltaic devices. Chem Commun 2012; 48:3686-3699.

[5••] Gokus T, Nair RR, Bonetti A, Böhmler M, Lombardo A, Novoselov KS, Geim AK, Ferrari AC, Hartschuh A. Making graphene luminescent by oxygen plasma treatment. ACS Nano 2009; 3:3963-3968. Band gap engineering in graphene. 
[6] Jiao L, Wang X, Diankov G, Wang H, Dai H. Facile synthesis of high-quality graphene nanoribbons. Nat Nanotechnol 2010; 5:321-325.

[7] Kelarakis A. From highly graphitic to amorphous carbon dots: a critical review. MRS Energy and Sustainability 2014; 1:E2.

[8] Yan X, Cui X, Li B, Li LS. Large, solution-processable graphene quantum dots as light absorbers for photovoltaics. Nano Lett 2010;10:1869-1873.

[9] Levesque I, Neabo JR, Rondeau-Gagne S, Vigier- Carriere C, Daigle M, Morin JF. Layered graphitic materials from a molecular precursor. Chem Sci 2014;5:831-836.

[10] Liu R, Wu D, Feng X, Mullen K. Bottom-up fabrication of photoluminescent graphene quantum dots with uniform morphology. J Am Chem Soc 2011;133:15221-15223.

[11] Lu J, Yeo PSE, Gan CK, Wu P, Loh KP. Transforming C 60 molecules into graphene quantum dots. Nat Nanotechnol 2011; 6:247-252.

[12•] Ye R, Xiang C, Lin J, Peng Z, Huang K, Yan Z, Cook N P, Samuel ELG, Hwang CC, Ruan G, Ceriotti G, Raji ARO, Martí, AA, Tour JM. Coal as an abundant source of graphene quantum dots. Nature Commun 2013;4:1-6. Release of high quality GQDs from inexpensive starting materials.

[13] Dong Y, Chen C, Zheng X, Gao L, Cui Z, Yang H, Guo C, Chi Y, Li CM. One-step and high yield simultaneous preparation of single- and multi-layer graphene quantum dots from CX-72 carbon black. J Mater Chem 2012; 22:8764-8766.

[14] Pan D, Zhang J, Li Z, Wu M. Hydrothermal route for cutting graphene sheets into blue luminescent graphene quantum dots. Adv Mater 2010; 22:734-738.

[15] Peng J, Gao W, Gupta BK, Liu Z, Romero-Aburto R, Ge L, Li Song, Alemany LB, Zhan X, Gao G, Vithayathil SA, Kaipparettu BA, Marti AA, Hayashi T, Zhu JJ, Ajayan PM. Graphene quantum dots derived from carbon fibers. Nano Lett 2012;12:844-849.

[16] Dong Y, Pang H, Ren S, Chen C, Chi Y, Yu T. Etching single-wall carbon nanotubes into green and yellow single-layer graphene quantum dots. Carbon 2013;64:245-251.

[17] Chua CK, Sofer Z, Simek P, Jankovsky O, Klımova K, Bakardjieva S, Kuckova SH, Pumera M. Synthesis of strongly fluorescent graphene quantum dots by cage-opening buckminsterfullerene. ACS Nano 2015;9:2548-2555. 
[18] Zhou JG, Booker C, Li R, Zhou X, Sham TK, Sun X, Ding Z. An electrochemical avenue to blue luminescent nanocrystals from multiwalled carbon nanotubes (MWCNTs). J Am Chem Soc 2007; 129:744-745.

[19] Zheng L, Chi Y, Dong Y, Lin J, Wang B. Electrochemiluminescence of water-soluble carbon nanocrystals released electrochemically from graphite. J Am Chem Soc 2009;131:4564-4565.

[20] Niyogi S, Bekyarova E, Itkis ME, Zhang H, Shepperd K, Hicks J, Sprinkle M, Berger C, Lau CN, de Heer WA, Conrad EH, Haddon RC. Spectroscopy of covalently functionalized graphene. Nano Lett 2010;10:4061-4066.

[21••] Chien CT, Li SS, Lai WJ, Yeh YC, Chen HA, Chen IS, Chen LC, Chen KH, Nemoto T, Isoda S, Chen M, Fujita T, Eda G, Yamaguchi H, Chhowalla M, Chen CW. Tunable photoluminescence from graphite oxide. Angew Chem Int Ed 2012;51:6662-6666. Important insights on the PL mechanism in GQDs.

[22] Robertson J, O' Reilly EP. Electronic and atomic structure of amorphous carbon. Phys Rev B 1987;35:2946-2957.

[23] Eda G, Lin YY, Mattevi C, Yamaguchi H, Chen HA, Chen IS, Chen CW, Chhowalla M. Blue photoluminescence from chemically derived graphene oxide. Adv Mater 2010;22:505 -509. [24] Li H, He X, Kang Z, Huang H, Liu Y, Liu J, Lian S, Tsang CHA, X. Yang X, Lee ST. Watersoluble fluorescent carbon quantum dots and photocatalyst design. Angew Chem Int Ed 2010;49:4430-4434.

[25] Kwon W, Kim YH, Lee CL, Lee M, Choi HC, T-W Lee TW, Rhee SW. Electroluminescence from graphene quantum dots prepared by amidative cutting of tattered graphite. Nano Lett 2014; 14:1306-1311.

[26•] Alam M, Ananthanarayanan A, Huang L, Lim KH, Chen P. Revealing the tunable photoluminescence properties of graphene quantum dots. J Mater Chem C 2014;2: 6954-6960. An interesting computational study on the PL performance of QGDs.

[27] Li LL, Ji J, Fei R, Wang CZ, Lu Q, Zhang JR, Jiang LP, Zhu JJ. A Facile Microwave avenue to electrochemiluminescent two-color graphene quantum dots. Adv Funct Mater 2012;22:29712979.

[28] Krysmann MJ, Kelarakis A, Giannelis EP. Photoluminescent carbogenic nanoparticles directly derived from crude biomass. Green Chem 2012; 14:3141-3145. 
[29] Sahu S, Behera B, Maiti TK, Mohapatra S. Simple one-step synthesis of highly luminescent carbon dots from orange juice: application as excellent bio-imaging agents. Chem Commun 2012; 48:8835-8837.

[30] Peng H, Travas-Sejdic J. Simple aqueous solution route to luminescent carbogenic dots from carbohydrates. Chem Mater 2009;21:5563-5565.

[31] Zhou L, He B, Huang J. Amphibious fluorescent carbon dots: one-step green synthesis and application for light-emitting polymer nanocomposites. Chem Commun 2013; 49: 8078-8080. [32] Jaiswal A, Ghosh SS, Chattopadhyay A. One step synthesis of C-dots by microwave mediated caramelization of poly(ethylene glycol). Chem Commun 2012; 48:407-409.

[33•] Cao L, Meziani MJ, Sahu S, Sun YP. Photoluminescence properties of graphene versus other carbon nanomaterials. Acc Chem Res 2013; 46:171-180. An in-depth critical discussion.

[34] Wang L, Zhu SJ, Wang HY, Qu SN, Zhang YL, Zhang JH, Chen QD, Xu HL, Han W, Yang B, Sun HB. Common origin of green luminescence in carbon nanodots and graphene quantum dots. ACS Nano 2014; 8:2541-2547.

[35] Cao L, Wang X, Meziani M J, Lu F, Wang H, Luo P G, Lin Y, Harruff BA, Veca LM, Murray D, Xie SY, Sun YP. Carbon dots for multiphoton bioimaging. J Am Chem Soc 2007; 129: 1131811319.

[36] Gupta V, Chaudhary N, Srivastava R, Sharma GD, Bhardwaj R, Chand S. Luminescent graphene quantum dots for organic photovoltaic devices. J Am Chem Soc 2011;133:9960- 9963. [37] Zhu C, Yang S, Wang G, Mo R, He P, Sun J, Di Z, Kang Z, Yuan N, Ding J, Ding G, Xiec $X$. A new mild, clean and highly efficient method for the preparation of graphene quantum dots without by-products. J Mater Chem B 2015;3: 6871-6876.

[38] Yang ST, Cao L, Luo PG, Lu F, Wang X, Wang H, Meziani MJ, Liu Y, Qi G, Sun YP. Carbon dots for optical imaging in vivo. J Am Chem Soc 2009; 131:11308-11309.

[39] Nurunnabi M, Khatun Z, Reeck GR, Lee DY, Lee Y. Near infra-red photoluminescent graphene nanoparticles greatly expand their use in noninvasive biomedical imaging. Chem Commun 2013;49:5079-5081.

[40] Li Y, Zhao Y, Cheng H , Hu Y, Shi G, Dai L, Qu L. Nitrogen-doped graphene quantum dots with oxygen-rich functional groups. J Am Chem Soc 2012;134:15-18. 
[41] Fei H, Ye R, Ye G, Gong Y, Peng Z, Fan X, Samuel ELG, Ajayan PM, Tour JM. Boron and nitrogen doped graphene quantum dots/graphene hybrid nanoplatelets as efficient electrocatalysts for oxygen reduction. ACS Nano 2014;8:10837-10843.

[42] Zhang HC, Ming H, Lian S, Huang H, Li H, Zhang L, Liu Y, Kang Z, Lee ST. $\mathrm{Fe}_{2} \mathrm{O}_{3}$ /carbon quantum dots complex photocatalysts and their enhanced photocatalytic activity under visible light. Dalton Trans 2011; 40:10822-10825.

[43] Zhang H, Huang H, Ming H, Li H, Zhang L, Liu Y, Kang Z. Carbon quantum dots/Ag $\mathrm{PO}_{4}$ complex photocatalysts with enhanced photocatalytic activity and stability under visible light. J Mater Chem 2012; 22:10501-10506.

[44] Sun H, Wu L, Wei W, Qu X. Recent advances in graphene quantum dots for sensing. Materials Today 2013;16:433-442.

[45] Fan L, Hu Y, Wang X, Zhang L, Li F, Han D, Li Z, Zhang Q, Wang Z, Niu L. Fluorescence resonance energy transfer quenching at the surface of graphene quantum dots for ultrasensitive detection of TNT. Talanta 2012;101:192-197.

[46] Bai JM, Zhang L, Liang RP, Qiu JD. Graphene quantum dots combined with europium ions as photoluminescent probes for phosphate sensing. Chem Eur J 2013;19:3822-3826.

[47] Zhanga YQ, Ma DK, Zhang YG, Chen W, Huang SM. N-doped carbon quantum dots for $\mathrm{TiO}_{2}$-based photocatalysts and dye-sensitized solar cells. Nano Energy 2013; 2:545-552.

[48] Li Y, Hu Y, Zhao Y, Shi G, Deng L, Hou Y, Qu L. An electrochemical venue to green luminescent graphene quantum dots as potential electron acceptors for photovoltaics. Adv Mater 2011;23:776-780.

[49] Kim JK, Park MJ, Kim SJ, Wang DH, Cho SP, Bae S, Park JH, Hong BH. Balancing light absorptivity and carrier conductivity of graphene quantum dots for high-efficiency bulk heterojunction solar cells. ACS Nano 2013;7:7207-7212.

- Of special interest.

•- Of outstanding interest. 